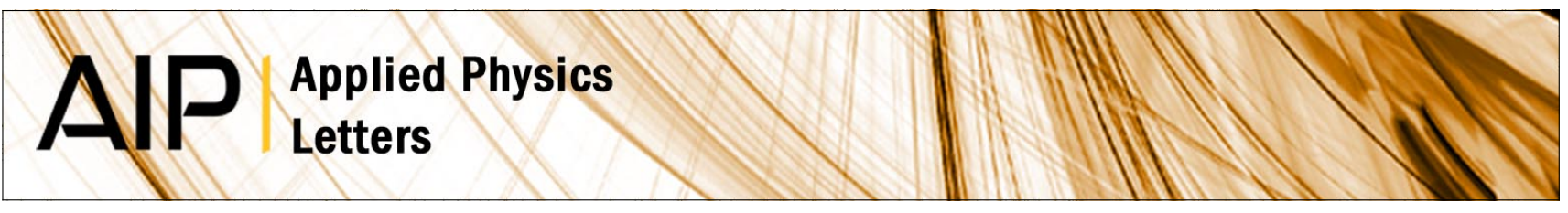

\title{
Easily monitored entangled state
}

M. A. Can, A. A. Klyachko, and A. S. Shumovsky

Citation: Appl. Phys. Lett. 81, 5072 (2002); doi: 10.1063/1.1521262

View online: http://dx.doi.org/10.1063/1.1521262

View Table of Contents: http://apl.aip.org/resource/1/APPLAB/v81/i26

Published by the American Institute of Physics.

Additional information on Appl. Phys. Lett.

Journal Homepage: http://apl.aip.org/

Journal Information: http://apl.aip.org/about/about_the_journal

Top downloads: http://apl.aip.org/features/most_downloaded

Information for Authors: http://apl.aip.org/authors

\section{ADVERTISEMENT}

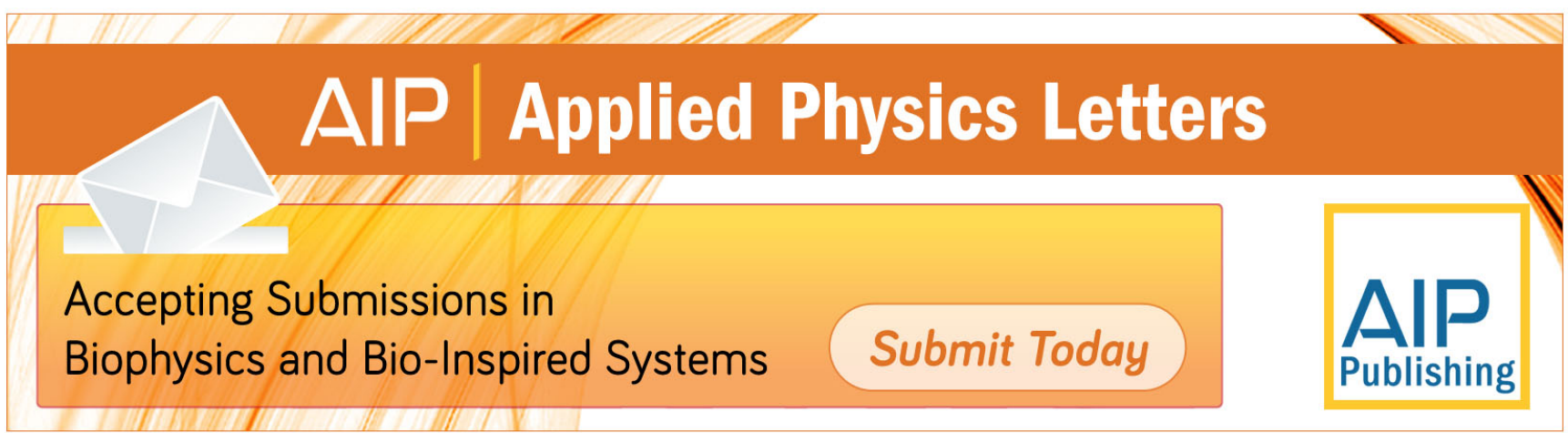




\title{
Easily monitored entangled state
}

\author{
M. A. Can, ${ }^{\text {a) }}$ A. A. Klyachko, and A. S. Shumovsky \\ Faculty of Science, Bilkent University, Bilkent, Ankara, 06533 Turkey
}

(Received 11 July 2002; accepted 20 September 2002)

We discuss the generation and monitoring of durable atomic entangled state via Raman-type process, which can be used in the quantum information processing. (C) 2002 American Institute of Physics. [DOI: 10.1063/1.1521262]

The problem of creating entangled states in atomic systems has attracted a great deal of interest (see Ref. 1 and references therein). In particular, the entangled states have been engineered through the use of cavity quantum electrodynamics ${ }^{2}$ and the technique of ion traps. ${ }^{3}$

An interesting proposal has been made recently ${ }^{4}$ (for further discussion, see Refs. 5 and 6). It was shown that a pure entangled state of two atoms in an optical resonator can be obtained through the exchange by a single photon. Since the excitation of the system either is carried by a cavity photon or is shared between the atoms, the absence of the photon leakage from the resonator can be associated with the presence of atomic entanglement. This entanglement can be observed in the process of continuous monitoring of the cavity decay. ${ }^{4}$ The importance of this scheme is caused by the fact that its realization seems to be easily available with present experimental technique. The result can also be generalized on the multi-atom systems. ${ }^{6}$

With respect to practical realization, it seems to be more convenient if the existence of atomic entanglement would manifest itself via a certain signal photon rather than via the absence of photons, as in Ref. 4. This implies that there should be at least two different modes interacting with the atoms such that the photon of one of them provides the correlation between the atoms, while the photon of the other mode can freely leave the resonator to signalize the rise of atomic entanglement.

In this letter we discuss a way to obtain a durable maximum entangled state of atoms in an optical resonator, which can be monitored through the detection of signal photons.

Consider the Raman-type process in a three-level atom shown in Fig. 1. Here $1 \leftrightarrow 2$ and $2 \leftrightarrow 3$ are the dipole transitions corresponding to the pump and Stokes modes, respectively, while the dipole transition between levels 1 and 3 is forbidden because of parity conservation. We assume that the two identical atoms of this type are located in a high-quality cavity tuned to resonance with $1 \leftrightarrow 2$ transition, while the Stokes photons can leak away freely (Fig. 2).

Assume that initially both atoms are in the ground state (level 1) and there is a single cavity photon, so that the initial state is

$$
\left|\psi_{0}\right\rangle=|1,1\rangle\left|1_{P}\right\rangle\left|V_{S}\right\rangle
$$

Here $\left|n_{P}\right\rangle$ denotes the $n$-photon state of the cavity (pump)

\footnotetext{
${ }^{a)}$ Electronic mail: can@fen.bilkent.edu.tr
}

mode and $\left|V_{S}\right\rangle$ denotes the vacuum state of the Stokes field. Then, the absorption of the cavity photon by atomic system should lead to the state

$$
\left|\psi_{1}\right\rangle=\frac{1}{\sqrt{2}}(|2,1\rangle+|1,2\rangle)\left|0_{P}\right\rangle\left|V_{S}\right\rangle,
$$

which manifests the entanglement of atoms excited to level 2. This atomic entanglement is similar to that discussed in Ref. 4 and has a very short lifetime, defined by the atom-field coupling constants for the allowed transitions. The decay of the excited atomic state (2) can either return the system into the initial state (1) or turn (2) into the state

$$
\left|\psi_{k}\right\rangle=\frac{1}{\sqrt{2}}(|3,1\rangle+|1,3\rangle)\left|0_{P}\right\rangle\left|1_{\mathrm{Sk}}\right\rangle,
$$

where $\left|n_{\mathrm{Sk}}\right\rangle$ denotes the state of $n$ Stokes photons with frequency $\omega_{\mathrm{Sk}}$. This state again manifests the maximum atomic entanglement. Since the cavity walls are supposed to be transparent for the Stokes photons and $3 \leftrightarrow 1$ is the dipoleforbidden transition, the atomic entanglement described by Eq. (3) would exist for a very long time, determined by the weak interaction between the atoms excited to level 3 and a certain dissipative environment. The creation of this atomic entanglement manifests itself by the Stokes photon that can be detected outside the cavity.

It should be noted that, in addition to $\left|\psi_{1}\right\rangle$ and $\left|\psi_{k}\right\rangle$, the following maximum entangled states

$$
\left|\phi_{1}\right\rangle=\frac{1}{\sqrt{2}}(|2,1\rangle-|1,2\rangle)\left|0_{P}\right\rangle\left|V_{S}\right\rangle,
$$

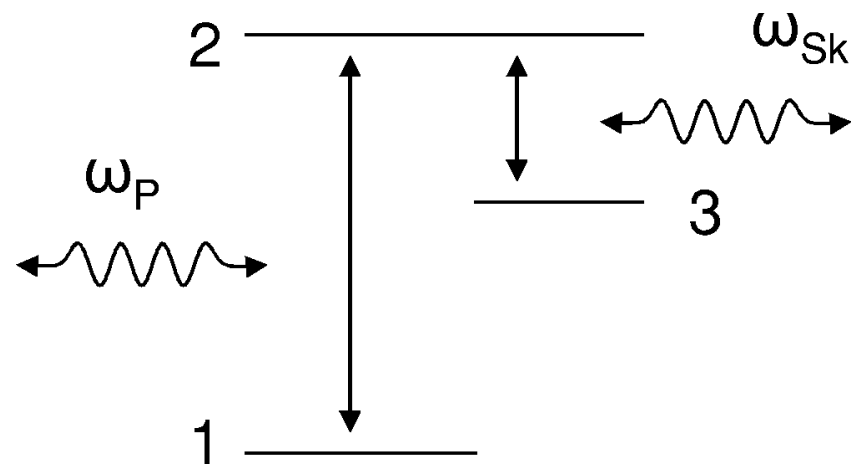

FIG. 1. Scheme of Raman-type process in an atom. Solid arrows show the allowed transitions. Wavy lines show the pump and Stokes photons. 


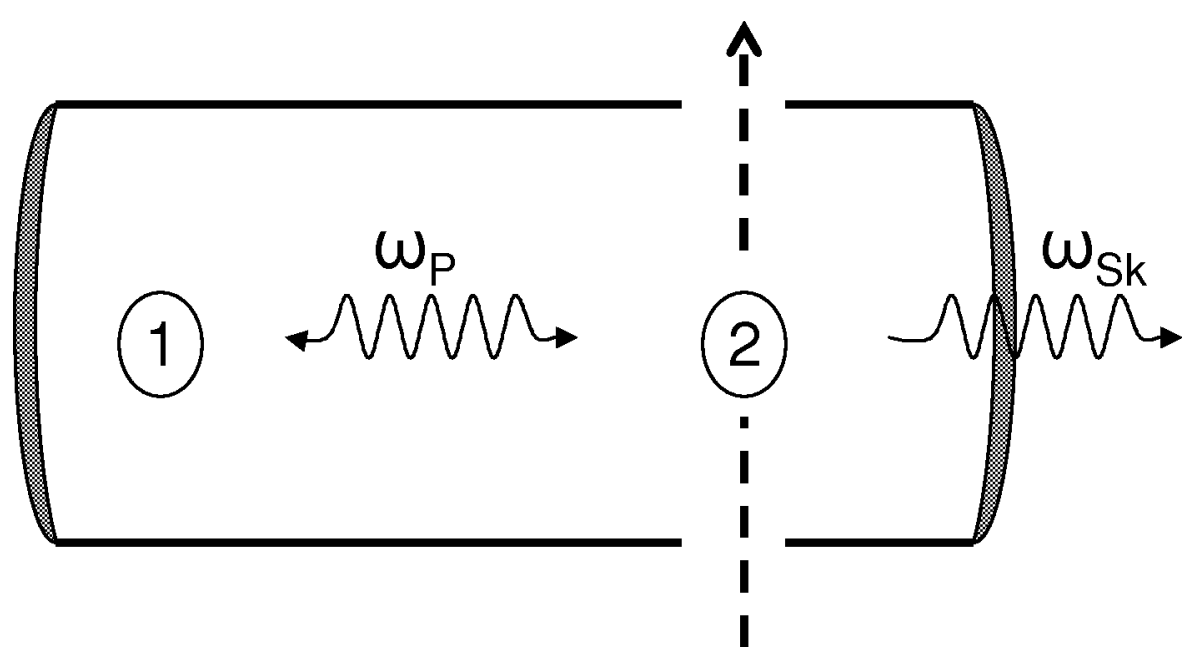

FIG. 2. Scheme of creation of a durable two-atom entanglement. Atom 1 is trapped in a cavity, while atom 2 can pass through the cavity. Wavy lines show the cavity and leaking out Stokes photons.

$$
\left|\phi_{k}\right\rangle=\frac{1}{\sqrt{2}}(|3,1\rangle-|1,3\rangle)\left|0_{P}\right\rangle\left|1_{\mathrm{Sk}}\right\rangle,
$$

also contribute into the base states of the system under consideration. Both of them are stable states, but they cannot be achieved in the process of evolution beginning with the initial state (1) (see Ref. 6). Therefore, they can be discarded.

To describe the quantum dynamics of the system, we note that the upper atomic level 2 can be adiabatically removed $^{7}$ (also see Ref. 8 and references therein). In this case, the two-photon transitions in effective two-level atoms described by the effective interaction Hamiltonian

$$
H_{\mathrm{int}}=\sum_{k} \sum_{f=1}^{2} \lambda_{k}\left\{R_{31}(f) a_{\mathrm{Sk}}^{+} a_{P}+H . c\right\}
$$

should be considered. Here $\lambda_{k}$ denotes an effective coupling constant has been defined in Ref. 7 and $R_{i j}(f)$ is the atomic operator corresponding to the transition $j \rightarrow i$ in the $f$ th atom. Under the influence of Eq. (4), the initial state (1) is directly transformed into (3), so that the intermediate entangled state (2) can be omitted. Then, the time-dependent wave function of the system takes the form

$$
|\Psi(t)\rangle=C_{0}(t)\left|\psi_{0}\right\rangle+\sum_{k} C_{k}(t)\left|\psi_{k}\right\rangle,
$$

where the time-dependent coefficients are defined by the Schrödinger equation together with the initial condition

$$
|\Psi(0)\rangle=\left|\psi_{0}\right\rangle, \quad C_{0}(0)=1, \quad C_{k}(0)=0 .
$$

Taking into account that the total Hamiltonian has the form

$$
\begin{aligned}
& H=H_{0}+H_{\mathrm{int}}, \\
& H_{0}=\omega_{P} a_{P}^{+} a_{P}+\sum_{k} \omega_{\mathrm{Sk}} a_{\mathrm{Sk}}^{+} a_{\mathrm{Sk}}+\omega_{31} \sum_{f=1}^{2} R_{33}(f),
\end{aligned}
$$

we get the following system of linear differential equations

$$
\begin{aligned}
& i \dot{C}_{0}=\omega_{P} C_{0}+\sum_{k} \lambda_{k} \sqrt{2} C_{k}, \\
& i \dot{C}_{k}=\left(\omega_{\mathrm{Sk}}+\omega_{31}\right) C_{k}+\lambda_{k} \sqrt{2} C_{0} .
\end{aligned}
$$

Here $\omega_{31}=E_{3}-E_{1}$ denotes the energy difference between levels 3 and 1 connected by the two-photon transition. These
Eqs. (7) together with the initial conditions (6) completely determine the evolution of the state (5). Using the standard methods, ${ }^{9}$ it is easy to show that the system evolves from the initial state (1) into the final state

$$
|\Psi(t)\rangle \rightarrow \sum_{k} J_{k}\left|\psi_{k}\right\rangle
$$

corresponding to the maximum atomic entanglement described by Eq. (3). Here

$$
J_{k}=\frac{-i \lambda_{k} \sqrt{2}}{\gamma / 2-i\left(\omega_{\mathrm{Sk}}+\omega_{31}-\omega_{P}-\Delta\right)},
$$

and

$$
\gamma=\left.2 \pi p\left(\omega_{\mathrm{Sk}}\right) \lambda_{k}^{2}\right|_{\omega_{\mathrm{Sk}}+\omega_{31}=\omega_{P}}
$$

is the parameter describing the rapidity of the exponential evolution to the entangled atomic state, $p\left(\omega_{k}\right)$ denotes the density of states corresponding to the Stokes field, and

$$
\Delta=-\mathcal{P}\left\{\int_{-\infty}^{\infty} \frac{p\left(\omega_{\mathrm{Sk}}\right) \lambda_{k}^{2} d \omega_{k}}{\omega_{k}+\omega_{31}-\omega_{P}}\right\}
$$

is a small frequency shift ( $\mathcal{P}$ denotes the principle value of the integral). Thus

$$
\begin{aligned}
|\Psi(t)\rangle= & e^{-\gamma t / 2} e^{-i\left(\omega_{P}-\Delta\right) t}\left|\psi_{0}\right\rangle \\
& -\sum_{k} \frac{i \lambda_{k} \sqrt{2}}{\gamma / 2-i\left(\omega_{\mathrm{Sk}}+\omega_{31}-\omega_{P}-\Delta\right)} \\
& \times\left[e^{-i\left(\omega_{\mathrm{Sk}}+\omega_{31}\right) t}-e^{-\gamma t / 2} e^{-i\left(\omega_{P}-\Delta\right) t}\right]\left|\psi_{k}\right\rangle,
\end{aligned}
$$

and the system evolves exponentially to the maximum entangled atomic state (3). In fact, this is a durable maximum entangled atomic state because the direct single-photon transition $3 \leftrightarrow 1$ is forbidden. The lifetime of this entangled state is defined by the slow nonradiative processes only.

Let us stress that the two advantages of the aboveconsidered three-level two-photon process in comparison with the previous scheme ${ }^{4,5}$ are, on the one hand, the durability of the entangled state, and, on the other hand, the simple monitoring of entanglement via detection of a Stokes photon. We think that the quantum information processing in the system under consideration can be arranged in the same way as in Ref. 10. 
The above long-life atomic entanglement can be interpreted as the long-distance entanglement as well within the following experimental scheme. Assume that one of the atoms is trapped in the cavity that supports a single-photon Fock state of the pump mode. The second atom passes through the cavity as shown in Fig. 2. Time of the propagation of the atom through the cavity, defined by the velocity of the atom, should be long enough to provide the preparing of the entangled state (3) with high probability. The creation of this state is signaled by detection of the Stokes photon. Thus, the measurement of the state of the moving atom at any distance from the cavity determines the state of the trapped atom.

Concerning the practical realization of the abovediscussed scheme, we should stress that the observation of single-atom Raman-type process in an optical cavity has been reported recently. ${ }^{11}$ In this work, the ${ }^{85} \mathrm{Rb}$ atom was used. The excited state 2 corresponds to $5 \mathrm{P}_{3 / 2}$ level, while the ground 1 and intermediate 3 states are the $5 S_{1 / 2}$ hyperfine levels separated by frequency $\omega_{31}=3 \mathrm{GHz}$, while Stokes field has the wavelength $\lambda_{S}=780 \mathrm{~nm}$. In this case, the lifetime of the state $|3\rangle$ is at least ten times longer than that for the excited state 2 .

Let us stress that the obtained result can be generalized to the multi-atom case in the same way as for the conventional single-photon process in two-level atoms. ${ }^{6}$ The increase of the number of atoms should lead to a speeding-up of the evolution to the entangled atomic state because of the Dicke-type process caused by the photon exchange between the atoms (see Ref. 12).
The authors would like to thank J. H. Eberly and P. L. Knight for useful discussions.

${ }^{1}$ A. Furusawa, J. L. Sörensen, S. L. Braunstein, C. A. Fuchs, H. J. Kimble, and E. S. Polzik, Science 282, 706 (1998); Advances in Atomic, Molecular, and Optical Physics, edited by B. Bederson and H. Walther (Academic, New York, 2000), Vol. 42; C. J. Myatt, B. E. King, Q. A. Turchette, C. A. Sackett, D. Kielpinski, W. H. Itano, C. Monroe, and D. J. Wineland, Nature (London) 403, 269 (2000); G. Rempe, Ann. Phys. (Leipzig) 9, 843 (2000); J. M. Raymond, M. Brune, and S. Haroche, Rev. Mod. Phys. 73, 565 (2001); B. Julsgaard, A. Kozhekin, and E. Polzik, Nature (London) 413, 400 (2001).

${ }^{2}$ E. Hagley, X. Maitre, G. Nogues, C. Wunderlich, M. Brune, J. M. Raymond, and S. Haroche, Phys. Rev. Lett. 79, 1 (1997).

${ }^{3}$ Q. A. Turchette, C. S. Wood, B. E. King, C. J. Myatt, D. Leibfried, W. M. Itano, C. Monroe, and D. J. Wineland, Phys. Rev. Lett. 81, 3631 (1998).

${ }^{4}$ M. B. Plenio, S. F. Huelga, A. Beige, and P. L. Knight, Phys. Rev. A 59, $2468(1999)$

${ }^{5}$ A. Beige, S. Bose, D. Braun, S. F. Huelga, P. L. Knight, M. B. Plenio, and V. Verdal, J. Mod. Opt. 47, 2583 (2000).

${ }^{6}$ M. A. Can, A. A. Klyachko, and A. S. Shumovsky, Phys. Rev. A 66, 022111 (2002)

${ }^{7}$ C. C. Gerry and J. H. Eberly, Phys. Rev. A 42, 6805 (1990).

${ }^{8}$ N. N. Bogolubov, Jr., F. Le Kien, and A. S. Shumovsky, Europhys. Lett. 4, 281 (1987); C. K. Law and J. H. Eberly, Phys. Rev. A 47, 3195 (1993); R. R. Puri, C. K. Law, and J. H. Eberly, Phys. Rev. A 50, 4212 (1994).

${ }^{9}$ C. W. Gardiner and P. Zoller, Quantum Noise (Springer, Berlin, 2000).

${ }^{10}$ S. Osnaghi, P. Bertet, A. Auffeves, P. Maioli, M. Brune, J. M. Raymond, and S. Haroche, Phys. Rev. Lett. 87, 037902 (2001).

${ }^{11}$ M. Hennrich, T. Legero, K. Khun, and G. Rempe, Phys. Rev. Lett. 85, 4872 (2000)

${ }^{12}$ A. S. Shumovsky and T. Kuang, in Interaction of Electromagnetic Field with Condensed Matter, edited by N. N. Bogolubov, A. S. Shumovsky, and V. I. Yukalov (World Scientific, Singapore, 1990). 